\title{
Correction to: On the Assessment of Security and Performance Bugs in Chromium Open-Source Project
}

\author{
Joseph Imseis, Costain Nachuma, Shaikh Arifuzzaman,
}

Minhaz Zibran, and Zakirul Alam Bhuiyan

\section{Correction to: \\ Chapter "On the Assessment of Security and Performance Bugs in Chromium Open-Source Project" \\ in: G. Wang et al. (Eds.): Dependability in Sensor, Cloud, and Big Data Systems and Applications, CCIS 1123, https://doi.org/10.1007/978-981-15-1304-6_12}

In the originally published version of the chapter 12 , the name of one of the contributors was mistakenly omitted. The authorship information has been corrected by adding the author Minhaz Zibran, affiliated with Computer Science Department at the University of New Orleans, Louisiana, USA. 